\title{
Rateless Space Time Block Code for Massive MIMO Systems
}

\author{
Ali H. Alqahtani, Ahmed Iyanda Sulyman, and Abdulhameed Alsanie \\ Department of Electrical Engineering, King Saud University, P.O. Box 800, Riyadh 11421, Saudi Arabia \\ Correspondence should be addressed to Ali H. Alqahtani; aqahtani@cti.edu.sa
}

Received 14 March 2014; Revised 25 June 2014; Accepted 1 July 2014; Published 3 August 2014

Academic Editor: Joonhyuk Kang

Copyright (C) 2014 Ali H. Alqahtani et al. This is an open access article distributed under the Creative Commons Attribution License, which permits unrestricted use, distribution, and reproduction in any medium, provided the original work is properly cited.

\begin{abstract}
This paper presents a rateless space time block code (RSTBC) for massive MIMO systems. The paper illustrates the basis of rateless space time codes deployments in massive MIMO transmissions over wireless erasure channels. In such channels, data may be lost or is not decodable at the receiver due to a variety of factors such as channel fading, interference, or antenna element failure. We show that RSTBC guarantees the reliability of the system in such cases, even when the data loss rate is $25 \%$ or more. In such a highly lossy channel, the conventional fixed-rate codes fail to perform well, particularly when channel state information is not available at the transmitter. Simulation results are provided to demonstrate the BER performance and the spectral efficiency of the proposed scheme.
\end{abstract}

\section{Introduction and Motivation}

Multiple-input multiple-output (MIMO) antenna systems have recently become one of the most significant technologies in modern wireless communications. MIMO wireless communication systems have attracted interests in both academy and industry, due to their ability to increase the systems reliability and capacity. The current fourth generation $(4 \mathrm{G})$ wireless standards, like LTE/LTE-A and WiFi (IEEE 802.11n), have used MIMO antenna systems to achieve significantly higher data rates and improve the spectral efficiency. The invention of MIMO system [1] led to the introduction of multiantenna coding scheme, known as space time code (STC) [2]. The scheme has the potential to significantly exploit MIMO gains offered by multiple antenna transmission, as well as coding gain offered by the encoding scheme that is deployed. A number of schemes employing multiple antenna arrays with STC were developed in [3-7]. Space time trellis code (STTC) and space time block code (STBC) are the two main types of STC techniques. Several recent wireless standards have used STBC, due to its low decoding complexity in comparison to STTC. As far as design criteria, it has been shown in [2] that the main code design criterion is the rank criterion, in which the rank of the difference of two distinct codewords has to be maximized. The codebook is said to be fully diverse, if this property is satisfied. Once the difference matrix has full rank, the product of its singular values is nonzero; then the second design criterion is maximizing the coding gain. Extensive work has been done on designing STCs possessing high gains $[8,9]$.

Moving forward to the fifth generation (5G) standards, MIMO techniques will continue to play a key role in further increasing the wireless data rates to the Gigabit range targeted. Demand for wireless communications is projected to grow rapidly over the next few years $[10,11]$. A potential technology for meeting this demand is massive MIMO systems (also known as large-scale antenna or large-scale MIMO systems). Massive MIMO is a promising technique for the next generation wireless systems as a multiuser multiple antenna system. The concept of massive MIMO involves the deployment of base stations with a very large number of antenna arrays, using perhaps hundreds of transceiver elements, to increase network capacity, improve reliability, and reduce the overall transmitted power. The distinguishing feature of massive MIMO is that a large number of antennas, possibly hundreds or even thousands, work together to deliver huge amount of data to multiple users on the same time-frequency resources. Moreover, large antenna arrays can focus the radiated energy precisely towards the intended receivers, thereby efficiently reducing interference. 
This improvement in performance also comes with accompanying enhancements in radiated energy efficiency. Although massive MIMO systems provide better spectral and energy efficiency compared with the existing MIMO systems, their deployments in cellular systems may come with new problems which arise from the difficulty of deploying a very large number of antenna elements and keeping them all in working condition continuously. This is an important practical deployment issue that has not yet been examined. There may arise situations whereby few antenna elements in the field have been damaged (or failed to work properly) and the data fed on to these elements is lost in the wireless transmission medium. This gives rise to the so-called wireless erasure channels.

In this work, we examine rateless coding techniques that can be used to solve this problem in the context of massive MIMO system, where a large number of antennas are employed for point-to-point as well as point-to-multipoint communications between a transmitter and a receiver. Unlike the conventional channel code, rateless code does not have a fixed rate, and most of the available work in the literature is an extension of the fountain codes over the erasure channels [12]. The first practical class of rateless codes is called LT (Luby transform) code, which was developed to the wellknown Raptor code [13]. For wireless channels, rateless code paradigm was addressed in many works such as [14-16]. A rateless code can operate "universally" over classes of channels and adapt its rate to the specific channel realization. The notion of rateless coding is conceptually similar to the hybrid automatic repeat request (HARQ) schemes [17], but the difference is mainly in the code construction where rateless codes can be considered as a continuous version of the HARQ schemes [14]. Theoretically speaking, based on the general notion of ratelessness, a rateless code generates a potentially infinite supply of encoding packets from the original data and sequentially transmits codeword symbols. Practically, rateless code has to be truncated to a finite number of blocks where each block has a certain likelihood of being decoded to recover the entire message. The receiver keeps trying to decode the message as it receives the codewords. When the receiver successfully decodes the message, it sends back an acknowledgement flag over a feedback channel to inform the transmitter to terminate the current codeword and start transmission of the next message.

To our knowledge, only few works have examined rateless space time codes [18]. Most of these works were conducted mainly over additive white Gaussian noise (AWGN) channel. There is not enough research work yet on rateless space time codes, even for the regular MIMO systems. For the case of massive MIMO systems, we are not aware of published work yet examining the combination of rateless STC with massive MIMO schemes. The characteristics of rateless codes and the erasure links that may arise due to failure of antenna elements in massive MIMO systems motivate us to propose the use of rateless coding technique in massive MIMO systems.

In this study, we examine the performance of RSTBC employed in massive MIMO systems. We illustrate the effectiveness of the scheme in combating lossy links that may arise during wireless transmissions. Our results show that

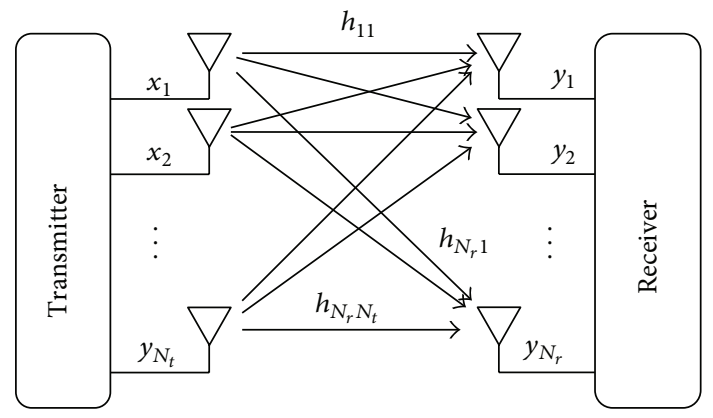

Figure 1: Basic MIMO system.

the proposed system can convert a lossy channel (such as a channel with $25 \%$-loss rate) into a lossless one and provide a reliable robust transmission when very large MIMO dimensions are used.

The remainder of the paper is organized as follows. Section 2 describes the system model and the basic problem formulation in our work. Performance evaluations of rateless STC over massive MIMO channels are presented in Section 3. Section 4 presents RSTBC for massive multiuser MIMO system. Simulation results for massive SU-MIMO and MUMIMO systems are presented and analyzed in Section 5. Finally, Section 6 presents the conclusion of the paper.

\section{System Model and Problem Formulation}

2.1. Conventional MIMO System. Consider a wireless communication system with $N_{t}$ transmit and $N_{r}$ receive antennas as shown in Figure 1. Assume that path gains between individual antenna pairs are independent and identically distributed (i.i.d.) Rayleigh-faded. This means that the maximal diversity gain is $N_{t} N_{r}$ which is equivalent to the total number of fading paths in the MIMO channel.

The output of the system can be expressed as

$$
\mathbf{Y}=\sqrt{\frac{E_{x}}{N_{o} N_{t}}} \mathbf{H X}_{C}+\mathbf{N}
$$

where $\mathbf{Y} \in \mathbb{C}^{N_{r} \times T}$ is the matrix of received signals over " $T$ " time slots, $\mathbf{H} \in \mathbb{C}^{N_{r} \times N_{t}}$ is the channel coefficients matrix whose elements are independent and identically distributed (i.i.d) complex Gaussian random variables (with zero mean and unit variance) given by

$$
\mathbf{H}=\left[\begin{array}{cccc}
h_{11} & h_{12} & \cdots & h_{1 N_{t}} \\
h_{21} & h_{22} & \cdots & h_{2 N_{t}} \\
\vdots & \vdots & \vdots & \vdots \\
h_{N_{r} 1} & h_{N_{r} 2} & \cdots & h_{N_{r} N_{t}}
\end{array}\right]
$$

and $E_{x}$ is the energy per symbol at each transmit antenna. The parameter $\mathbf{X}_{C} \in \mathbb{C}^{N_{t} \times T}$ denote the space time code matrix, while $\mathbf{N} \in \mathbb{C}^{N_{r} \times T}$ is the Gaussian noise matrix (with i.i.d. zero mean and unit variance complex Gaussian elements). 


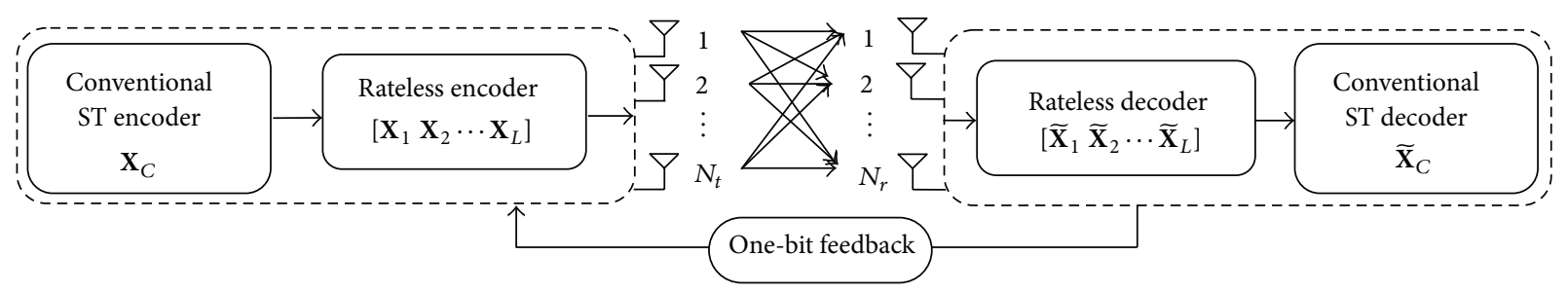

FIGURE 2: Sequential rateless space time block coded MIMO system.

2.2. Lossy Channel Model. In this work, we assume a lossy channel in which losses are introduced in the channel transfer matrix $\mathbf{H}$ as follows:

$$
\begin{aligned}
\mathbf{H} \odot \mathbf{D}= & {\left[\begin{array}{cccc}
h_{11} & h_{12} & \cdots & h_{1 N_{t}} \\
h_{21} & h_{22} & \cdots & h_{2 N_{t}} \\
\vdots & \vdots & \vdots & \vdots \\
h_{N_{r} 1} & h_{N_{r} 2} & \cdots & h_{N_{r} N_{t}}
\end{array}\right] } \\
& \odot\left[\begin{array}{cccc}
d_{11} & d_{12} & \cdots & d_{1 N_{t}} \\
d_{21} & d_{22} & \cdots & d_{2 N_{t}} \\
\vdots & \vdots & \vdots & \vdots \\
d_{N_{r} 1} & d_{N_{r} 2} & \cdots & d_{N_{r} N_{t}}
\end{array}\right],
\end{aligned}
$$

where $\mathbf{D} \in \mathbb{C}^{N_{r} \times N_{t}}$ is a matrix of random binary numbers representing losses experienced during transmission (i.e., the dropped links, where zeros represent losses and ones represent lossless links), and $\odot$ denotes the elementwise multiplication operation (Hadamard product). Losses may occur when some transmitted symbols are dropped, especially in high volume downloading through broadcasting over noisy wireless networks, or when some transmitter components, such as antenna elements, fail to work. Furthermore, interference from all other transmit antenna elements is considered at the receiver.

2.3. Sequential Rateless STBC. The purpose of this subsection is to introduce the mechanism of RSTBC as depicted by the block diagram in Figure 2. In this system, each message is encoded into a codeword of $L$ blocks in which each block takes $T$ channel use where $T$ is sufficiently large and the channel coherence time remains constant for the entire codeword length (i.e., up to $L$ blocks) [16]. Then, the system input-output relationship is expressed as

$$
\begin{aligned}
& {\left[\begin{array}{llll}
\mathbf{Y}_{1} & \mathbf{Y}_{2} & \cdots & \mathbf{Y}_{L}
\end{array}\right]=\sqrt{\frac{E_{x}}{L N_{t} N_{o}}}(\mathbf{H} \odot \mathbf{D})} \\
& \times\left[\begin{array}{llll}
\mathbf{X}_{1} & \mathbf{X}_{2} & \cdots & \mathbf{X}_{L}
\end{array}\right] \\
& +\left[\begin{array}{llll}
\mathbf{N}_{1} & \mathbf{N}_{2} & \cdots & \mathbf{N}_{L}
\end{array}\right],
\end{aligned}
$$

where $\mathbf{X}_{l} \in \mathbb{C}^{N_{t} \times T}(l=1, \ldots, L)$ is the codeword matrix transmitted during the $l$ th block, $\mathbf{H} \in \mathbb{C}^{N_{r} \times N_{t}}$ is the channel transfer matrix, $\mathbf{N}_{l} \in \mathbb{C}^{N_{r} \times T}$ is a noise matrix with i.i.d. zero mean and unit variance complex Gaussian elements, and $\mathbf{Y}_{l} \in \mathbb{C}^{N_{r} \times T}$ is the output signals matrix. The conventional space time codeword matrix $\mathbf{X}_{\mathbf{C}}$ is encoded into a rateless ST codeword matrix $\left[\begin{array}{lllll}\mathbf{X}_{1} & \mathbf{X}_{2} & \cdots & \mathbf{X}_{L}\end{array}\right]$, where $\mathbf{X}_{l}$, for example, when $T=2$, can be constructed as

$$
\mathbf{X}_{l}=\left[\begin{array}{cc}
x_{1} & x_{2}^{*} \\
x_{2} & -x_{1}^{*} \\
\vdots & \vdots \\
x_{N_{t}-1} & x_{N_{t}}^{*} \\
x_{N_{t}} & -x_{N_{t}-1}^{*}
\end{array}\right]
$$

A power constraint on $\mathbf{X}_{l}$ is introduced as

$$
E\left[\frac{1}{T}\left\|\mathbf{X}_{l}\right\|_{F}^{2}\right] \leq N_{t} \quad \text { for } l=1, \ldots, L
$$

where $E[\cdot]$ denotes the expected value.

At the receiving end, it is desired to decode a transmitted message of mutual information $\delta$ with the codeword $\left[\begin{array}{llll}\mathbf{X}_{1} & \mathbf{X}_{2} & \cdots & \mathbf{X}_{l}\end{array}\right]$. Let $I$ denote the mutual information after receiving the codeword block $\mathbf{X}_{l}$. If $I<\mathcal{S}$, the receiver remains silent and waits for the next block. This process continues until the receiver collects the sufficient number of blocks that is required to recover the message. When $I \geq \mathcal{S}$ after the $l$ th block, the receiver decodes the received codeword $\left[\begin{array}{llll}\widetilde{\mathbf{X}}_{1} & \widetilde{\mathbf{X}}_{2} & \ldots & \widetilde{\mathbf{X}}_{l}\end{array}\right]$ and sends one-bit feedback to the transmitter to stop transmitting the remaining part of the current codeword and start transmitting the next message immediately [16]. For the MIMO channel model considered in Figure 2, which we call the lossy channel, the decoding process is conducted sequentially, first using $\widetilde{\mathbf{X}}_{1}$, then $\left[\begin{array}{ll}\widetilde{\mathbf{X}}_{1} & \widetilde{\mathbf{X}}_{2}\end{array}\right]$ if $\widetilde{\mathbf{X}}_{1}$ is not sufficient, and so forth. As a result, to achieve high efficiency, sequential RSTBC requires large delays. Since many applications are more sensitive to delay characteristics, we develop fast RSTBC for massive MIMO channels as described in the following subsection.

2.4. Rateless STBC for Massive SU-MIMO System. In this subsection, we present RSTBC in massive single-user MIMO (SU-MIMO) systems. As we have explained, in the rateless coding concept discussed earlier, the codewords $\left\{\mathbf{X}_{l}\right\}, l=$ $1,2, \ldots, L$, are transmitted through different channels that are orthogonal in time. Therefore, sequential RSTBC requires time delay to achieve reliability of the transmission. This delay constraint disallows coding over a large number of blocks. Fortunately, with massive MIMO configurations, in which a lot of antennas are used, the large number of antenna elements available can be exploited to overcome this problem. This can be accomplished by transmitting the entire rateless 


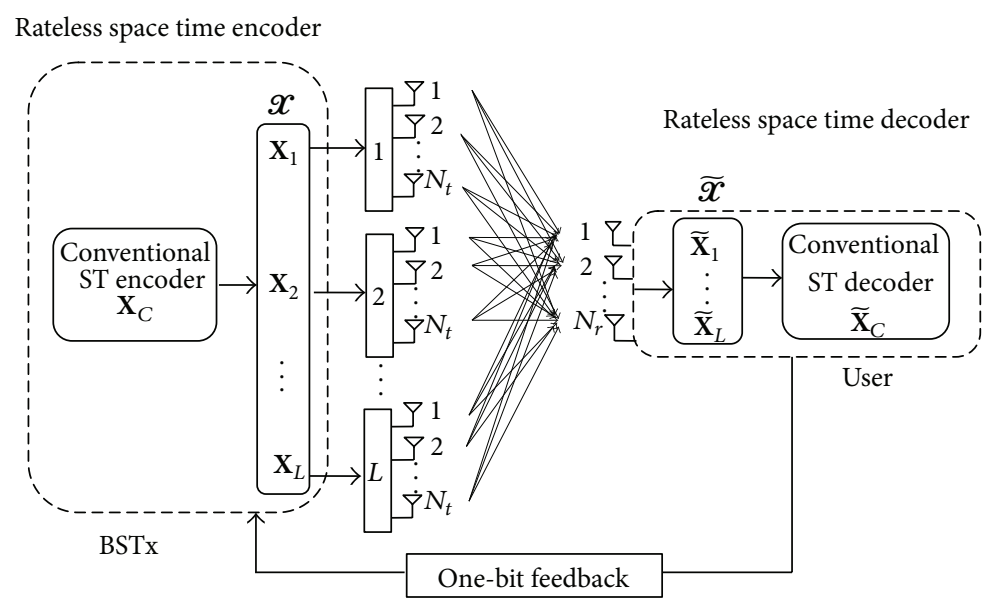

FIGURE 3: Rateless space time block code for single-user massive MIMO system.

codeword $\left[\begin{array}{llll}\mathbf{X}_{1} & \mathbf{X}_{2} & \cdots & \mathbf{X}_{L}\end{array}\right]$ simultaneously through different antenna elements. Thus, fast and reliable transmission is achieved.

Now, we consider a downlink massive SU-MIMO shown in Figure 3. In this scenario, a base station transmitter (BSTx) is equipped with a large number of antennas. We assume that the user is equipped with $N_{r}$ receiving antennas. The channel is assumed to have lossy characteristics. The input-output relationship can be expressed as

$$
\boldsymbol{U}=\sqrt{\frac{E_{x}}{L N_{t} N_{o}}}(\mathscr{H} \odot \mathscr{D}) \mathscr{X}+\mathscr{N} \text {. }
$$

In this equation, $\boldsymbol{U} \in \mathbb{C}^{N_{r} \times T}$ is the output matrix; $\mathscr{X}=$ $\left[\begin{array}{lll}\mathbf{X}_{1} & \cdots & \mathbf{X}_{L}\end{array}\right]^{T} \in \mathbb{C}^{L N_{t} \times T}$ is the codeword transmitted matrix; $\mathscr{N} \in \mathbb{C}^{N_{r} \times T}$ is the noise matrix; $\mathscr{H} \in \mathbb{C}^{N_{r} \times L N_{t}}$ is the channel transfer matrix; and $\mathscr{D} \in \mathbb{C}^{N_{r} \times L N_{t}}$ is a matrix of random binary numbers representing losses experienced during transmission (i.e., the dropped links, where zeros represent losses and ones represent reception), and each element in $\mathscr{D}$ at time $i$ is denoted by $d_{m n}^{i}$ (where $m=$ $1,2, \ldots, N_{r}$ and $\left.n=1,2, \ldots, L N_{t}\right)$. We impose a power constraint on $\mathscr{X}$ so that

$$
E\left[\frac{1}{T}\|\mathscr{X}\|_{F}^{2}\right] \leq L N_{t}
$$

The block diagram in Figure 3 depicts the system of (7) in which the BSTx contains large number of antenna elements which are grouped into $L$ groups (each group contains $N_{t}$ transmit antennas) and the rateless codeword $\mathscr{X}$ is transmitted through $L N_{t}$ transmit antennas over $T$ time slots. The user receiver contains $N_{r}$ receive antennas, where $L N_{t}>N_{r}$ so that the system is considered as $L N_{t} \times N_{r}$ massive SU-MIMO system. The number of antennas selected to transmit $\mathscr{X}$ depends on the number of blocks used in rateless coding.

To illustrate how the system shown in Figure 3 works, at the BSTx, first, the encoder generates $L$ redundant blocks $\mathscr{X}=\left[\begin{array}{lll}\mathbf{X}_{1} & \cdots & \mathbf{X}_{L}\end{array}\right]^{T}$, and then $L$ antenna groups (each of
$N_{t}$ antenna elements) are selected to transmit $\mathscr{X}$ simultaneously using a total of $L N_{t}$ transmit antennas through lossy channel $\mathscr{H}$. The number of blocks that need to be collected by the user depends on channel conditions; that is, a more lossy channel needs more redundant blocks. Once the underlying message is decoded completely, a feedback is sent from the user to the BSTx to terminate the current transmission and then move on to transmit the next message. Otherwise, the receiver waits for another return of transmission where the number of blocks $L$ is increased and, consequently, the number of selected groups is increased. As we stated before, the BSTx is equipped with a very large number of antennas perhaps hundreds or thousands, so the process continues until a reliable reception is achieved. The receiver collects as many redundancy blocks as needed to decode the message and corresponding received matrix $\boldsymbol{U}$ is used to estimate the transmitted codeword as $\widetilde{\mathscr{X}}=\left[\begin{array}{lll}\widetilde{\mathbf{X}}_{1} & \ldots & \widetilde{\mathbf{X}}_{L}\end{array}\right]^{T}$. The received signal by the user at the $m$ th receiving antenna at time $i$ is expressed in (9), and the maximum likelihood (ML) estimate $\widetilde{\mathscr{X}}$ is given by (10).

\section{Performance Limits and Design Criterion}

In this section, we discuss the performance of rateless codes employed in massive MIMO systems and provide limits on the rates and reliability achieved. In addition, design criterion of rateless codes is briefly described.

3.1. Probability of Error Performance. Consider the system depicted in Figure 3, if $L$ blocks are used at the BSTx; then the received signal by the user at the $m$ th receiving antenna at time $i$ can be expressed as

$$
\begin{array}{r}
u_{m}^{i}=\sqrt{\frac{E_{x}}{L N_{t} N_{o}}} \sum_{n=1}^{L N_{t}} h_{m n}^{i} d_{m n}^{i} x_{n}^{i}+n_{m}^{i}, \\
m=1,2, \ldots, N_{r} .
\end{array}
$$


At time $i, x_{n}^{i}$ is the signal transmitted by the $n$th antenna where $n=1,2, \ldots, L N_{t} ; h_{m n}^{i}$ is the channel coefficient from a transmitting antenna $n$ to a receiving antenna $m$; and $n_{m}^{i}$ is the noise at the $m$ th receiving antenna. Also, losses are introduced by $d_{m n}^{i} \in\{0,1\}$ where $d_{m n}^{i}=0$ when there is a loss, and $d_{m n}^{i}=1$ when there is no loss. Throughout this analysis, flat Rayleigh fading channel coefficients are assumed.

For decoding, a maximum likelihood (ML) estimate of the transmitted codeword $\widetilde{\mathscr{X}}$ is computed as

$$
\widetilde{\mathscr{X}}=\arg \min _{\mathscr{X}}\left\|\boldsymbol{U}-\sqrt{\frac{E_{x}}{L N_{o} N_{t}}}(\mathscr{H} \odot \mathscr{D}) \mathscr{X}\right\|_{F}^{2},
$$

where $\|\cdot\|_{F}^{2}$ denotes the squared Frobenius norm. The conditional probability of transmitting the codeword $\mathscr{X}$ and deciding erroneously in favor of $\overline{\mathscr{X}}$ at the decoder is the pairwise error probability (PWEP) given by

$$
P(\mathscr{X} \longrightarrow \widetilde{\mathscr{X}} \mid \mathscr{H}) \leq Q\left(\sqrt{\frac{E_{x}}{2 L N_{t} N_{o}} d_{E}^{2}(\mathscr{X}, \widetilde{\mathscr{X}})}\right),
$$

where $Q$ denotes the $Q$ function and $d_{E}^{2}(\mathscr{X}, \widetilde{\mathscr{X}})$ denotes the Euclidean distance between the transmitted rateless space time codeword matrix $\mathscr{X}$ and the erroneously received codeword matrix $\widetilde{\mathscr{X}}$ can be expressed as

$$
\begin{aligned}
d_{E}^{2}(\mathscr{X}, \widetilde{\mathscr{X}}) & =\|(\mathscr{H} \odot \mathbf{D})(\mathscr{X}-\widetilde{\mathscr{X}})\|_{F}^{2} \\
& =\sum_{i=1}^{T} \sum_{m=1}^{N_{r}}\left|\sum_{n=1}^{L N_{t}} h_{m n}^{i} d_{m n}^{i}\left(x_{n}^{i}-\widetilde{x}_{n}^{i}\right)\right|^{2} .
\end{aligned}
$$

Invoking the Chernoff bound, $Q(x) \leq(1 / 2) e^{-\left(x^{2} / 2\right)}$, the PWEP in (11) is upper-bounded as

$$
\begin{aligned}
& P(\mathscr{X} \longrightarrow \widetilde{\mathscr{X}} \mid \mathscr{H}) \\
& \quad \leq \frac{1}{2} \exp \left(-\frac{E_{x}}{4 L N_{t} N_{o}}\|(\mathscr{H} \odot \mathscr{D})(\mathscr{X}-\widetilde{\mathscr{X}})\|_{F}^{2}\right) .
\end{aligned}
$$

Let us define a codeword difference matrix $\mathscr{B}=\mathscr{X}-\widetilde{\mathscr{X}}$ and an $L N_{t} \times L N_{t}$ codeword distance matrix $\mathscr{A}=\mathscr{B} \cdot \mathscr{B}^{H}$. Then we can use the singular value decomposition (SVD) theorem to obtain

$$
\mathscr{A}=\mathrm{V}^{H} \cdot \mathbf{\Lambda} \cdot \mathrm{V}
$$

where $(\cdot)^{H}$ denotes the Hermitian transpose of a matrix; $\mathbf{V}$ is a unitary matrix with orthogonal eigenvector columns, and $\boldsymbol{\Lambda}$ is a diagonal matrix of eigenvalues $\left\{\lambda_{n}\right\}_{n=1}^{L N_{t}}$. By assuming slow Rayleigh fading channel, the channel gains do not change during $T$ symbol periods, and hence the time index $i$ can be omitted. If we denote the $(m, n)$ th element of $\mathscr{H} \mathbf{V}$ by $\Omega_{m n}$, then the Euclidean distance can be rewritten as

$$
\|(\mathscr{H} \odot \mathscr{D}) \mathscr{B}\|_{F}^{2}=\sum_{m=1}^{N_{r}} \sum_{n=1}^{L N_{t}} \lambda_{n} d_{m n}\left|\Omega_{m n}\right|^{2} .
$$

From (15), it is easy to observe that when there are losses (due to broken links for example), the Euclidean distance becomes smaller compared to the case without losses since $d_{m n}=0$ when there is a loss, and $d_{m n}=1$ when there is no loss. Consequently, the PWEP expression in (13) becomes large as the loss rate increases. To compensate for this loss, rateless code is applied by using $L$ redundant blocks. Thus, the expression in (15) becomes large as $L$ increases, thereby enhancing the PWEP performance.

3.2. Rate and Reliability Performance. For our proposed scheme, we next examine the rate and reliability achieved which promotes better insight into the performance of the system. For conventional systems, let $R$ denote the average number of bits transmitted per channel use, while $r$ denotes the normalized (with respect to SNR) rate expressed as [19]

$$
r=\lim _{\mathrm{SNR} \rightarrow \infty} \frac{R}{\log _{2}(\mathrm{SNR})} .
$$

Here, the parameter $r$ is also referred to as the spatialmultiplexing gain [20]. For a given $r$, let the probability of error be denoted as $P_{e}(r)$; then the diversity gain $d(r)$ which is a measure of the system reliability achieved can be obtained as [19]

$$
d(r)=-\lim _{\mathrm{SNR} \rightarrow \infty} \frac{\log _{2} P_{e}(r)}{\log _{2}(\mathrm{SNR})} .
$$

Now, for rateless coding with $L$ blocks, the maximum possible value of the diversity gain $d^{*}(r)$ at each $r$ is given by $[16,17]$

$$
d^{*}(r)=f\left(\frac{r}{L}\right), \quad 0 \leq r \leq \min \left\{L N_{t}, N_{r}\right\},
$$

where $f(\cdot)$ is the piecewise linear function joining the points $\left(k,\left(L N_{t}-k\right)\left(N_{r}-k\right)\right)$ for integral $k=0, \ldots, \min \left\{L N_{t}, N_{r}\right\}$. Equation (18) is based on the tradeoff between the diversity and spatial-multiplexing gains (DMG), developed by Zheng and Tse in [19], and gives a type of the diversity-multiplexingdelay (DMD) tradeoff introduced in [17]. This type of tradeoff between diversity and multiplexing gains is a function of the maximum number of blocks $L$ used in rateless coding to recover the original message. In addition, for sequential RSTBC, the maximum delay is $L T$ transmission slots before being able to decode the transmitted message, while massive MIMO system depicted in Figure 3 utilizes the maximum number of transmit antennas as $L N_{t}$ over $T$ time slots.

Next, let $R_{l}$ be the expected value of the realized rate (in bits per channel use) for each transmitted rateless codeword $\mathrm{X}_{l}$; then $R_{l}$ can be expressed as

$$
R_{l}=\frac{L}{l} R, \quad l=1,2, \ldots, L .
$$

Thus, compared with conventional coding schemes that have rate $R$, the rateless code achieves multiple rate levels $(R,(L /(L-1)) R,(L /(L-2)) R, \ldots, L R)$. In doing so, 


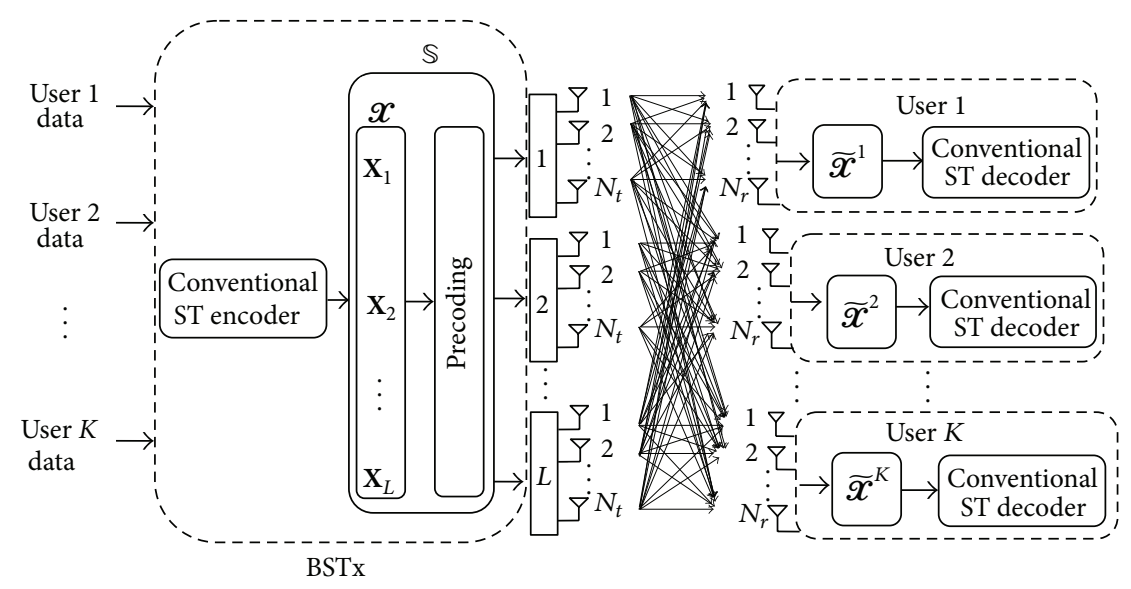

FIGURE 4: Rateless space time block code for massive multiuser MIMO system.

the effective multiplexing gain of the system can be expressed as [16]

$$
r_{e}=\lim _{\mathrm{SNR} \rightarrow \infty} \frac{R_{l}}{\log _{2}(\mathrm{SNR})}
$$

As a result, rateless codes also have multiple multiplexing gains as well. The diversity-multiplexing tradeoff (DMT) can then be used to analyze the systems performance for all possible transmission rates, providing performance limits for rateless codes.

3.3. Design Criterion for Rateless Codes. Generally speaking, rank criterion and determinant criterion are two different criteria for space time code design $[2,3]$. The classical code design criterion for the i.i.d. Rayleigh fading channel is the determinant criterion. However, the code design criterion for rateless codes is quite different from the determinant criterion, even though this criterion is also somewhat involved. It was shown in [16] that the design of rateless codes for MIMO channels follows the principle of approximately universal codes introduced in [21]. The approximately universal criterion ensures that the highest error event probability decays exponentially with SNR given any fading channel. This can be ignored when decoding any $l$ th subset codeword $\left\{\mathbf{X}_{l}\right\}$ in the set of all nonoutage events.

\section{RSTBC for Massive Multiuser MIMO System}

Consider a downlink massive multiuser MIMO (MUMIMO) system shown in Figure 4. In this scenario, a BSTx, equipped with a large number of antennas, communicates simultaneously with $K$ independent users on the same timefrequency resources where each user devise has $N_{r}$ receiving antennas. The channel is assumed to be uncorrelated and the channel state information (CSI) is known at both the transmitter and receiver. The channel matrix $\mathbb{U}$ can be written as

$$
\mathbb{H}=\left[\begin{array}{c}
\mathscr{H}_{1} \\
\mathscr{H}_{2} \\
\vdots \\
\mathscr{H}_{k}
\end{array}\right]
$$

where $\mathscr{H}_{k} \in \mathbb{C}^{N_{r} \times L N_{t}}, k=1,2, \ldots, K$, is the channel matrix corresponding to the $k$ th user. In order to eliminate the effects of the multiuser interference (MUI) at the specific receiving antennas, zero forcing ( $\mathrm{ZF}$ ) precoding is applied at the BSTx with a precoding matrix $\mathbf{G}$ given by [22]

$$
\mathbf{G}=\beta \mathscr{H}_{k}^{H}\left(\mathscr{H}_{k} \mathscr{H}_{k}^{H}\right)^{-1},
$$

where $(\cdot)^{H}$ denotes the conjugate transpose and $\beta$ is a normalized factor given by

$$
\beta=\sqrt{\frac{L N_{t}}{\operatorname{tr}\left[\left(\mathscr{H}_{k} \mathscr{H}_{k}^{H}\right)^{-1}\right]}},
$$

where $\operatorname{tr}[\cdot]$ denotes the trace of a matrix.

Let $\mathscr{X} \in \mathbb{C}^{K L N_{t} \times T}$ represents the RSTBC matrix constructed at the BSTx for all users over " $T$ " time slots. After the transmitter precoding operations, the resulting transmitted matrix $\mathbb{S} \in \mathbb{C}^{L N_{t} \times T}$ can be written as

$$
\mathbb{S}=\mathrm{G} \mathscr{X}
$$

where $\mathbf{G} \in \mathbb{C}^{L N_{t} \times K L N_{t}}$ is the precoding matrix expressed in (22). The received matrix $\bigvee \in \mathbb{C}^{K N_{r} \times T}$ may be written as

$$
\mathbb{Y}=\sqrt{\frac{E_{x}}{L N_{t} N_{o}}}(\mathbb{H} \odot \mathbb{D}) \mathbb{S}+\mathbb{W},
$$


where $\mathbb{S} \in \mathbb{C}^{L N_{t} \times T}$ is the codeword transmitted matrix; $\mathbb{W} \in \mathbb{C}^{K N_{r} \times T}$ is the noise matrix; $\mathbb{U} \in \mathbb{C}^{K N_{r} \times L N_{t}}$ is the channel transfer matrix; and $\mathbb{D} \in \mathbb{C}^{K N_{r} \times L N_{t}}$ is a matrix of random binary numbers representing losses experienced during transmission as stated earlier. Figure 4 depicts RSTBC employed in massive MU-MIMO system.

Now, let the notation $s_{k}^{m}$ indicates the transmitted symbol to the $k$ th user, and the symbol is precoded at the BSTx such that the fading-free data is received at the $m$ th antenna of the user, $m=1,2, \ldots, N_{r}$; then the received symbol by the $k$ th user can be described as

$$
y_{k}= \begin{cases}\beta s_{k}^{j}+w_{k}^{j}, & \text { if } j=m, \\ v_{k}^{j}+w_{k}^{j}, & \text { if } j \neq m,\end{cases}
$$

where $w_{k}^{j}$ denotes the Gaussian noise for the $k$ th user at the $j$ th receiving antenna, and $v_{k}^{j}=\sum_{n}^{L N_{t}} h_{k}^{j n} \widehat{\mathcal{s}}, j \neq m$, is the effect of channel fading and multiuser interference. For user $k$, the ML detection operation can be expressed as

$$
\widetilde{\mathbb{S}}_{k}^{j}=\arg \min _{\mathbb{S}}\left\|\frac{1}{\beta} \mathbb{Y}_{k}-\mathbb{S}_{k}^{j}\right\|_{F}^{2},
$$

where $\widetilde{\mathbb{S}}_{k}^{j}$ is the estimated matrix at the $j$ th antenna of the $k$ th user.

Although, we used precoding to cancel the interference, lossy channel disallows perfect interference cancellation. Therefore, MUI cannot be completely eliminated whenever lossy links are encountered in the data transmission, and hence this should be considered in massive MIMO systems. When losses occur, the precoding matrix expressed in (22) will be affected. Consequently, Euclidian distance given by (27) will be smaller compared to the case without losses, and then the performance of the system degrades. Introducing losses effects in (26), the received symbol by the $k$ th user can now be rewritten as

$$
y_{k}= \begin{cases}\beta s_{k}^{j}+w_{k}^{j}+z_{k}^{j}, & \text { if } j=m, \\ \sum_{n} h_{k}^{j n} \widehat{s}+w_{k}^{j}, & \text { if } j \neq m,\end{cases}
$$

where $z_{k}^{j}$ represents the residual interference caused by the lossy channel for the $k$ th user at the $j$ th receiving antenna, due to the fact that the precoding matrix at BSTx is unable to perfectly remove MUI in a lossy link (since the losses in transmission are unknown a priori to the precoder at the BSTx). We show later by simulation that RSTBC with a large number of blocks can adequately combat the effect of this interference from lossy links.

\section{Simulation Results and Discussion}

In this section, we present simulation results for the bit error rate (BER) performance and the corresponding spectral efficiency (in $\mathrm{b} / \mathrm{s} / \mathrm{Hz}$ ) of RSTBC utilized in MIMO scheme. In the simulations, we considered QPSK and 16-QAM modulation format. It is assumed that the fading coefficients from each transmit antenna to the receiver are mutually

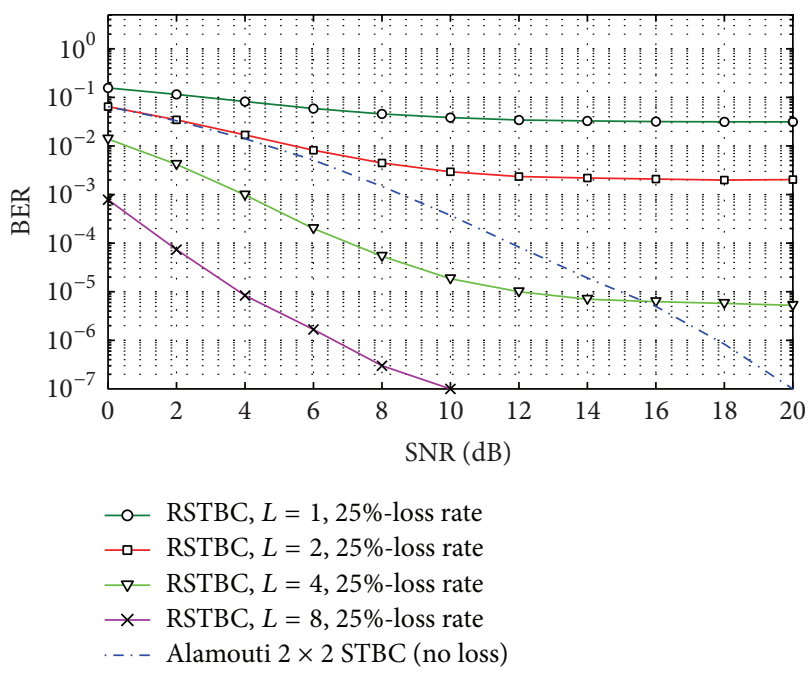

FIGURE 5: BER of RSTBC $(L=1,2,4$, and 8$)$ for MIMO system with QPSK and 25\%-loss rate $\left(N_{t}=2, N_{r}=2, T=2\right)$.

uncorrelated and Rayleigh-distributed. It is also assumed that the receiver has perfect knowledge of the channel. First, simulation results of sequential RSTBC depicted in Figure 2 are presented in Subsection 5.1. Next, the results of RSTBC for the massive SU-MIMO system follow in Subsection 5.2. Finally, Subsection 5.3 introduces the results of RSTBC for the massive MU-MIMO system.

5.1. Simulation Results of Sequential RSTBC. First, to illustrate the behavior of RSTBC for various numbers of blocks, we present the BER of $2 \times 2$ MIMO system with QPSK modulation employing RSTBC in Figure 5. The simulation results for RSTBC (with $L=1,2,4$, and 8 ) in a 25\%-loss rate channel are shown in comparison with the conventional Alamouti scheme. As indicated from the figure, it is obvious that when the number of used blocks, $L$, in RSTBC increases, the BER performance gets better gradually due to compensation introduced by RSTBC for the losses. As a result, the original data can be recovered even when losses occurred. Additionally, it is inferred from the figure that, for each value of $L$, the BER approaches a fixed value at high SNR because RSTBC, with the current number of blocks, is no longer able to compensate for further losses. Therefore, it is required to increase $L$ to achieve enhancement until all losses are eliminated. At such point in time, one can claim that a lossy channel has been made to become like the conventional system (without losses) by employing RSTBC. Figure 6 illustrates the corresponding spectral efficiency where the maximum value of $2 \mathrm{~b} / \mathrm{s} / \mathrm{Hz}$ for QPSK is achieved, as expected.

5.2. Simulation Results of RSTBC for Massive SU-MIMO System. Next, we consider the massive SU-MIMO system depicted in Figure 3. We have tested RSTBC for various number of blocks ( $L=1,2,4,8,16$, and 32) applied on various massive MIMO configurations based on the block diagram 


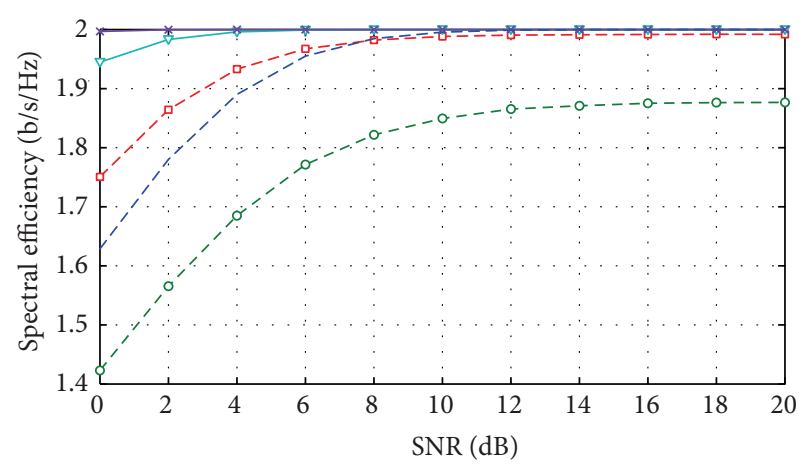

$$
\begin{aligned}
& - \text { - } \text { RSTBC, } L=1,25 \% \text {-loss rate } \\
& -\square-\text { RSTBC, } L=2,25 \% \text {-loss rate } \\
& -\nabla-\text { RSTBC, } L=4,25 \% \text {-loss rate } \\
& -* \text { RSTBC, } L=8,25 \% \text {-loss rate } \\
& --- \text { Alamouti } 2 \times 2 \text { STBC (no loss) }
\end{aligned}
$$

FIGURE 6: Spectral efficiency of RSTBC with $(L=1,2,4$, and 8$)$ for MIMO system with QPSK and 25\%-loss rate $\left(N_{t}=2, N_{r}=2, T=\right.$ 2).

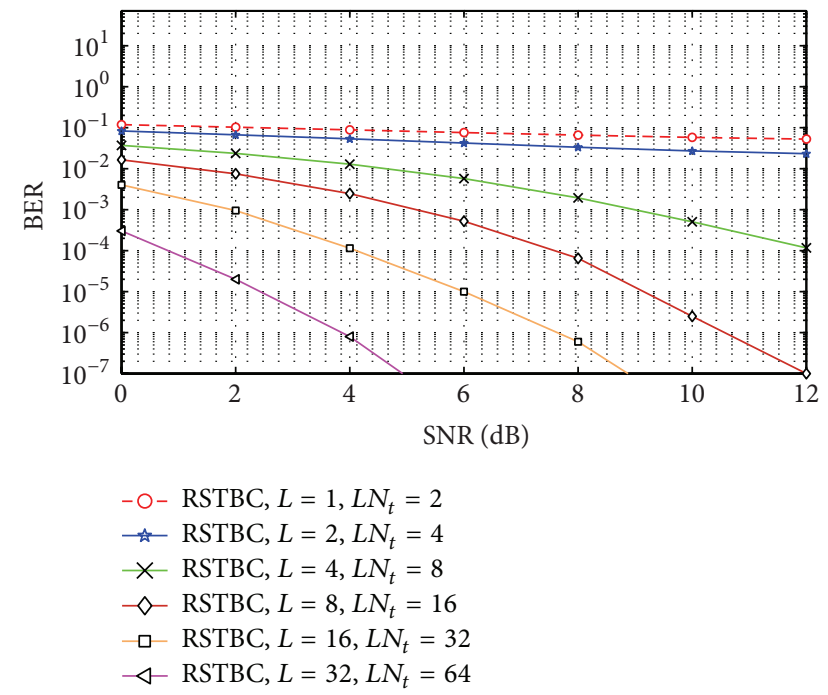

FIGURE 7: BER of RSTBC for massive SU-MIMO up to $L N_{t}=64$ with 16-QAM for 25\%-loss rate.

depicted in Figure 3 for 16-QAM modulation format. The channel is assumed to have a $25 \%$-loss rate.

As shown in Figure 7, it is observed that, by utilizing RSTBC, a massive MIMO system with $L N_{t}=64$ is able to convert a $25 \%$-loss rate channel into an approximately lossless one, as the error floor in the BER curve vanishes at this massive antenna configuration. This is a very good result for massive antenna systems. Developers have been trying to find different ways to utilize the large degrees of freedom available in a massive MIMO system. We demonstrate in this simulation that RSTBC with $L=32$ is capable of protecting the wireless transmission system against failures in antenna elements and other losses that may arise up to $25 \%$. The corresponding spectral efficiency is shown in Figure 8 where RSTBC approaches $4 \mathrm{~b} / \mathrm{s} / \mathrm{Hz}$ when $16-\mathrm{QAM}$ is used. It is

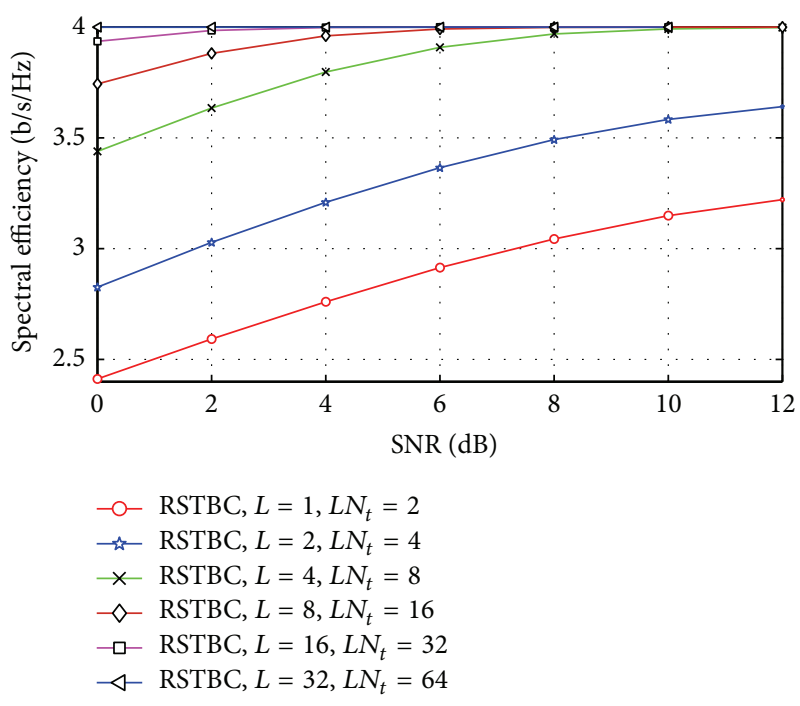

FIGURE 8: Spectral efficiency of RSTBC for massive SU-MIMO up to $L N_{t}=64$ with 16-QAM for 25\%-loss rate.

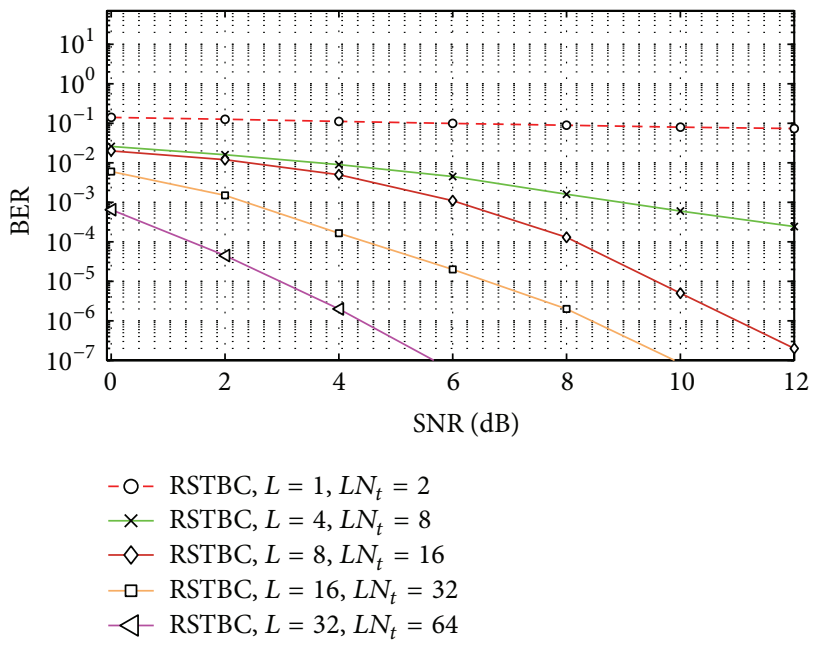

Figure 9: BER of RSTBC for massive MU-MIMO up to $L N_{t}=64$ ( $K=2$ users) with 16-QAM for 25\%-loss rate.

clear from the figure that RSTBC with $L=32$ effectively improves the efficiency of lossy systems. Therefore, it is shown that the proposed RSTBC, when deployed in such massive MIMO systems, exhibits a capacity-achieving characteristic and effectively combats the problem of lossy links. Table 1 illustrates the BER performance and spectral efficiency (at SNR $=4 \mathrm{~dB}$ ) as inferred from Figures 7 and 8, respectively.

5.3. Simulation Results of RSTBC for Massive MU-MIMO System. In this section, we present simulation results from the BER performance of RSTBC for massive MU-MIMO system described by Figure 4. The BER curves are averaged over all users. In the simulation, we considered 16-QAM modulation format. Figure 9 shows the BER performance of a multiuser MIMO system employing RSTBC. In this figure, we considered 2 users, each with $N_{r}=2$, where the channel 
TABLE 1: BER and spectral efficiency of RSTBC in massive SUMIMO with 16-QAM at SNR $=4 \mathrm{~dB}$ and loss rate $=25 \%$.

\begin{tabular}{lccc}
\hline$L$ & $L N_{t}$ & BER, $\times 10^{-3}$ & Spectral efficiency, b/s/Hz \\
\hline 1 & 2 & 100 & 2.8 \\
2 & 4 & 54 & 3.2 \\
4 & 8 & 13 & 3.79 \\
8 & 16 & 2.5 & 3.96 \\
16 & 32 & 0.1 & 4 \\
32 & 64 & 0.0008 & 4 \\
\hline
\end{tabular}

loss rate is $25 \%$. The RSTBC is applied with different number of blocks ( $L=1,4,8,16$, and 32). As shown in the figure, the BER performance gets better when the number of blocks in RSTBC increases, due to the compensation introduced. Hence, we have shown that the RSTBC can be applied to achieve transmission reliability in massive MIMO systems with lossy channels. We note also that the BER curve (for each simulation parameter $L, L N_{t}$ ) is slightly degraded compared to the corresponding case in the SU-MIMO system shown in Figure 7, as expected.

\section{Conclusion}

This work proposed the use of rateless space time block code (RSTBC) in massive MIMO systems and examined the BER performance and spectral efficiency of the scheme. Massive MIMO systems employ large numbers of antenna elements. Therefore, it is probable that when deployed practically on base stations, frequent cases of antenna element failure may arise resulting in a lossy channel model. A rateless space time code can be used to protect data transmission in such situations and still guarantee the reliability of the system. We conclude that it is possible to recover the original data from a certain amount of encoded data even when the losses are up to $25 \%$, using RSTBC with very large MIMO dimensions.

\section{Conflict of Interests}

The authors declare that there is no conflict of interests regarding the publication of this paper.

\section{Acknowledgment}

This work was supported by the NSTIP Strategic Technologies Programs at King Saud University (no. 11-ELE1880-02) in the Kingdom of Saudi Arabia.

\section{References}

[1] G. J. Foschini and M. J. Gans, "On limits of wireless communications in a fading environment when using multiple antennas," Wireless Personal Communications, vol. 6, no. 3, pp. 311-335, 1998.

[2] V. Tarokh, N. Seshadri, and A. R. Calderbank, "Space-time codes for high data rate wireless communication: performance criterion and code construction," IEEE Transactions on Information Theory, vol. 44, no. 2, pp. 744-765, 1998.
[3] S. M. Alamouti, "A simple transmit diversity technique for wireless communications," IEEE Journal on Selected Areas in Communications, vol. 16, no. 8, pp. 1451-1458, 1998.

[4] G. J. Foschini, "Layered space-time architecture for wireless communication in a fading environment when using multielement antennas," Bell Labs Technical Journal, vol. 1, no. 2, pp. 41-59, 1996.

[5] J. Guey, M. P. Fitz, M. R. Bell, and W. Kuo, "Signal design for transmitter diversity wireless communication systems over Rayleigh fading channels," in Proceedings of the IEEE 46th Vehicular Technology Conference, pp. 136-140, May 1996.

[6] N. Dao and C. Tellambura, "Semiorthogonal space-time block codes," IEEE Transactions on Information Theory, vol. 56, no. 1, pp. 168-180, 2010.

[7] V. Tarokh, H. Jafarkhani, and A. R. Calderbank, "Space-time block codes from orthogonal designs," IEEE Transactions on Information Theory, vol. 45, no. 5, pp. 1456-1467, 1999.

[8] F. Oggier, G. Rekaya, J. Belfiore, and E. Viterbo, "Perfect spacetime block codes," IEEE Transactions on Information Theory, vol. 52, no. 9, pp. 3885-3902, 2006.

[9] K. P. Srinath and B. S. Rajan, "Improved perfect space-time block codes," IEEE Transactions on Information Theory, vol. 59, no. 12, pp. 7927-7935, 2013.

[10] T. L. Marzetta, G. Caire, M. Debbah, I. Chih-Lin, and S. K. Mohammed, "Special issue on massive MIMO," Journal of Communications and Networks, vol. 15, no. 4, pp. 333-337, 2013.

[11] E. Larsson, O. Edfors, F. Tufvesson, and T. Marzetta, "Massive MIMO for next generation wireless systems," IEEE Communications Magazine, vol. 52, no. 2, pp. 186-195, 2014.

[12] J. Byers, M. Luby, M. Mitzenmacher, and A. Rege, "A digital fountain approach to reliable distribution of bulk data," in Proceedings of the ACM Conference on Applications, Technologies, Architectures, and Protocols for Computer Communication (SIGCOMM '98), pp. 56-67, Vancouver, Canada, September 1998.

[13] A. Shokrollahi, "Raptor codes," IEEE Transactions on Information Theory, vol. 52, no. 6, pp. 2551-2567, 2006.

[14] J. Castura and Y. Mao, "Rateless coding over fading channels," IEEE Communications Letters, vol. 10, no. 1, pp. 46-48, 2006.

[15] M. M. Shanechi, U. Erez, and G. W. Wornell, "Rateless codes for MIMO channels," in Proceedings of the IEEE Global Telecommunications Conference (GLOBECOM '08), pp. 1-5, New Orleans, La, USA, December 2008.

[16] F. Yijia, L. Lifeng, E. Erkip, and H. V. Poor, "Rateless coding for MIMO fading channels: performance limits and code construction," IEEE Transactions on Wireless Communications, vol. 9, no. 4, pp. 1288-1292, 2010.

[17] H. El Gamal, G. Caire, and M. O. Damen, "The MIMO ARQ channel: diversity-multiplexing-delay tradeoff," IEEE Transactions on Information Theory, vol. 52, no. 8, pp. 3601-3621, 2006.

[18] U. Erez, G. W. Wornell, and M. D. Trott, "Rateless space-time coding," in Proceedings of the IEEE International Symposium on Information Theory (ISIT '05), pp. 1937-1941, Adelaide, Australia, September 2005.

[19] L. Zheng and D. N. C. Tse, "Diversity and multiplexing: a fundamental tradeoff in multiple-antenna channels," IEEE Transactions on Information Theory, vol. 49, no. 5, pp. 1073-1096, 2003.

[20] S. A. Pawar, K. R. Kumar, P. Elia, P. V. Kumar, and B. A. Sethuraman, "Space-time codes achieving the DMD tradeoff of the MIMO-ARQ channel," IEEE Transactions on Information Theory, vol. 55, no. 7, pp. 3101-3114, 2009. 
[21] S. Tavildar and P. Viswanath, "Approximately universal codes over slow-fading channels," IEEE Transactions on Information Theory, vol. 52, no. 7, pp. 3233-3258, 2006.

[22] K. M. Humadi, A. I. Sulyman, and A. Alsanie, "Spatial modulation concept for massive multiuser MIMO systems," International Journal of Antennas and Propagation, vol. 2014, Article ID 563273, 9 pages, 2014. 

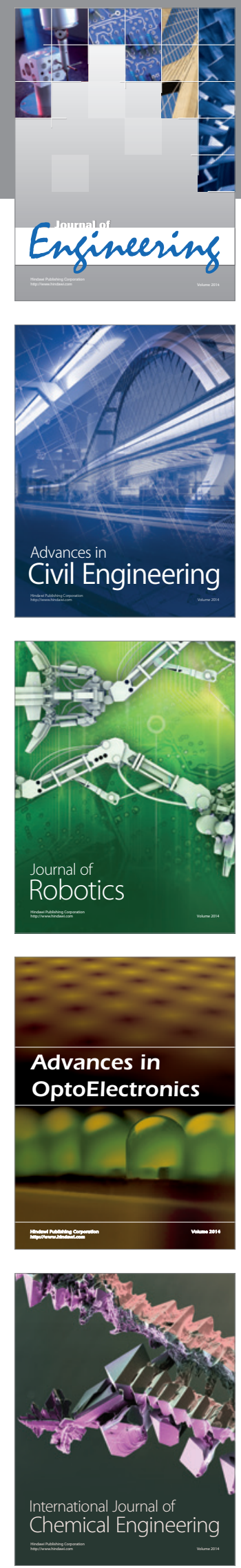

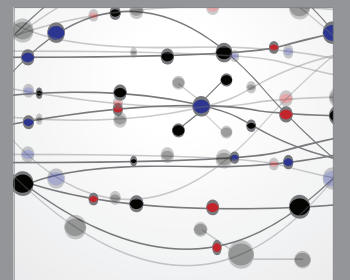

The Scientific World Journal
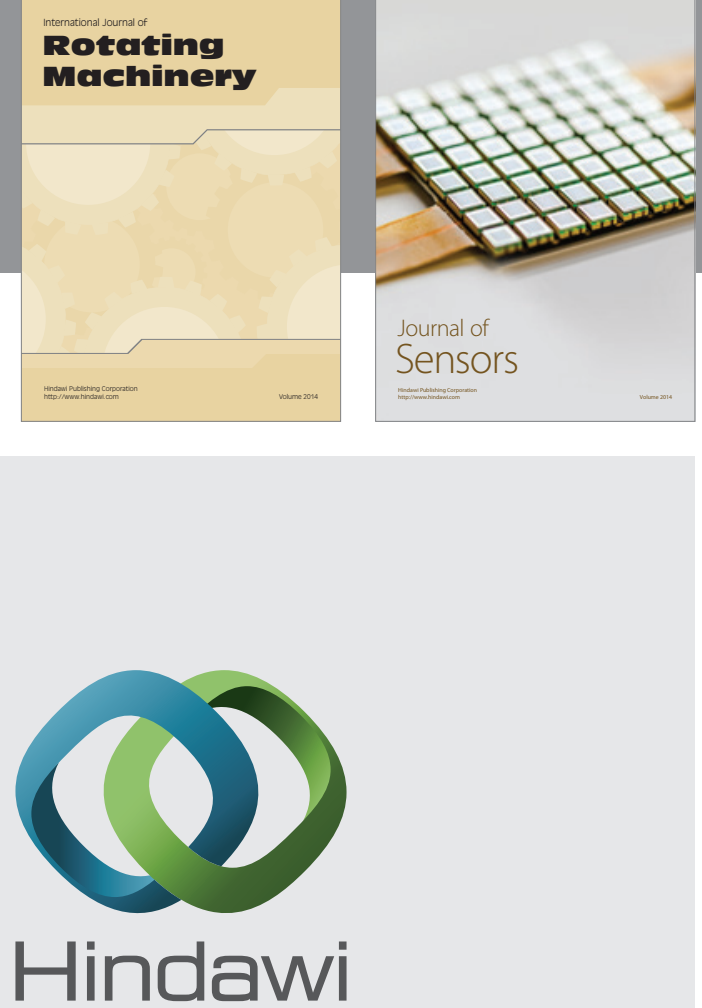

Submit your manuscripts at http://www.hindawi.com
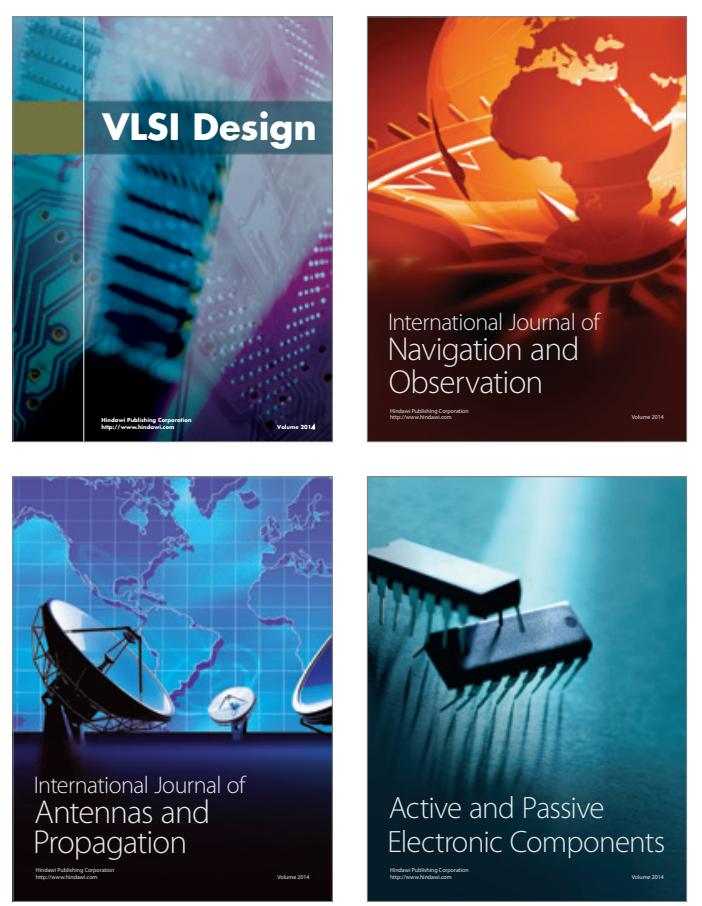
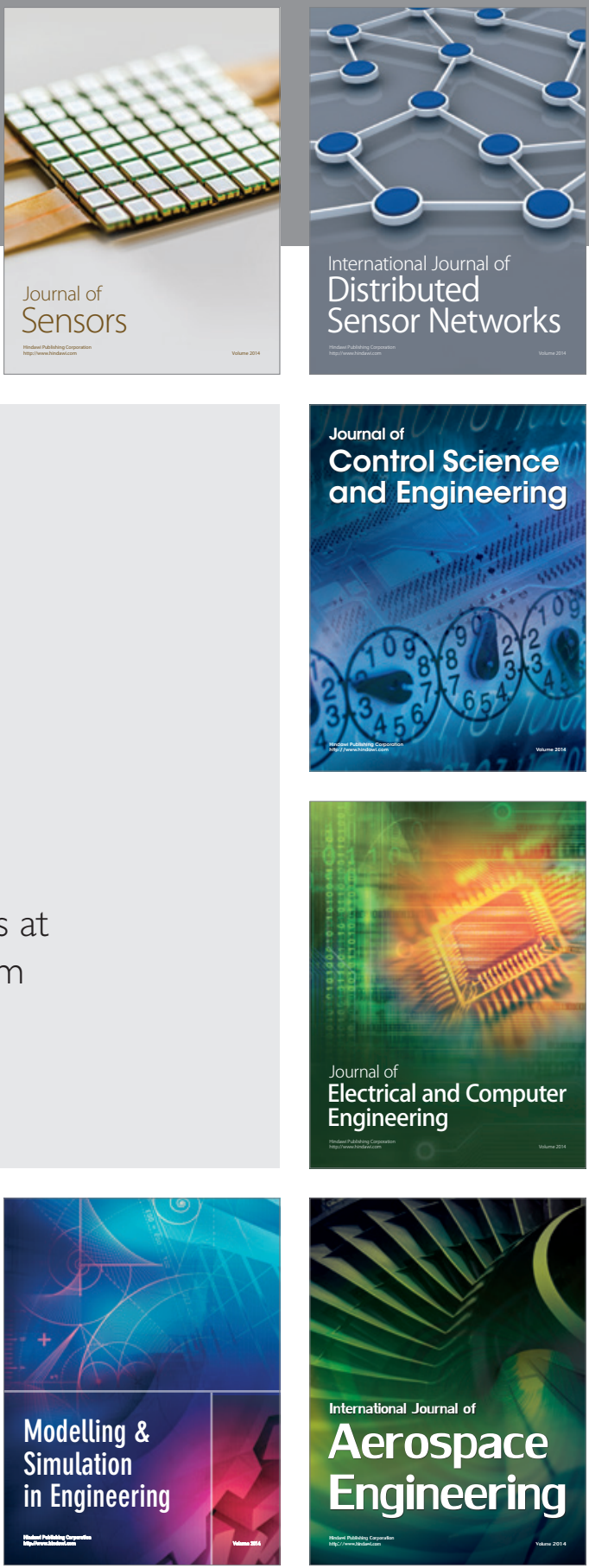

Journal of

Control Science

and Engineering
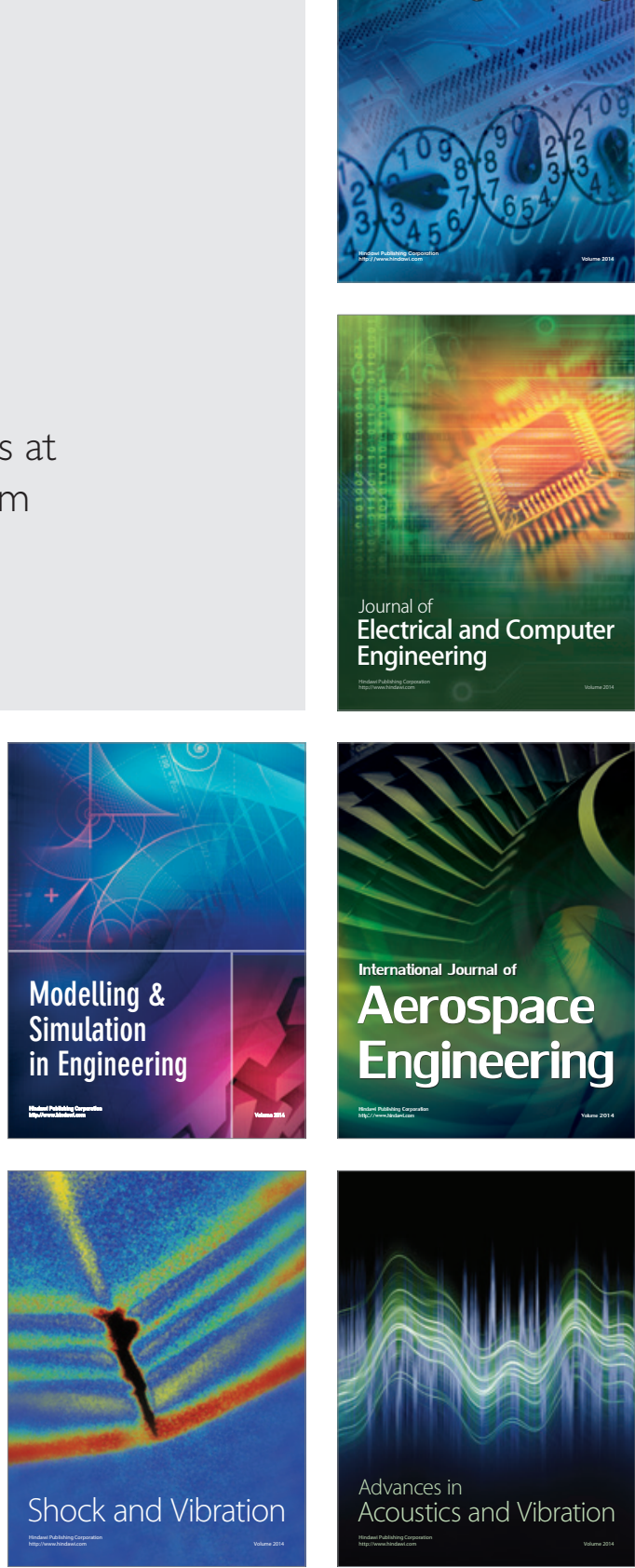